\title{
APERTURE DEPENDENCE OF 'RHIC CELLS \\ ON MULTIPOLE FIELDS IN THE \\ DEDICATED RHIC DIPOLES
}

G. F. De11

(Received June 1,1984 ) 
RHIC-PG-S7

Aperture Dependence of BHIC CELLS ON MULTI POLE FIELDS IN THE DEDICATED RHIC DIPOLES

$$
\text { GIF. DELL }
$$

(Received June 1, 1984) 
APERTURE DEPENDENCE OF RHIC CELLS ON MULTIPOLE FIELDS IN THE

DEDICATED RHIC DIPOLES

G.F: DeII

Abstract: Effects of multipoles in the dedicated RHIC dipoles have been studied by tracking particles through a lattice consisting of 81 RHIC cells. The dynamic aperture with no magnetic multipoles is greater than $59 \mathrm{~mm}$ at $\Delta \mathrm{P} / \mathrm{P}=0$. Inclusion of magnet multipoles reduces the dynamic aperture to $\sim 29 \mathrm{~mm}$. When a $30 \mathrm{~mm}$ aperture test is used, the beam size in the dipoles is limited to $27 \mathrm{~mm}$.

A lattice consisting of 81 . RHIC cells ( $2 \pi$ bend) has been used to evaluate the effects of systematic multipoles in the "dedicated" RHIC dipoles. A half cell is $14.80 \mathrm{~m}$ long and contains one $10.7 \mathrm{~m}$ dipole centered between the quadrupoles; the length of which are $1.9 \mathrm{~m}$. A chromaticity correcting sextupole and a multipole element (SF and MF or SD and MD) are located at the center of each quadrupole, while dipoles have a multipole element MB at each end: Fig. 1 .

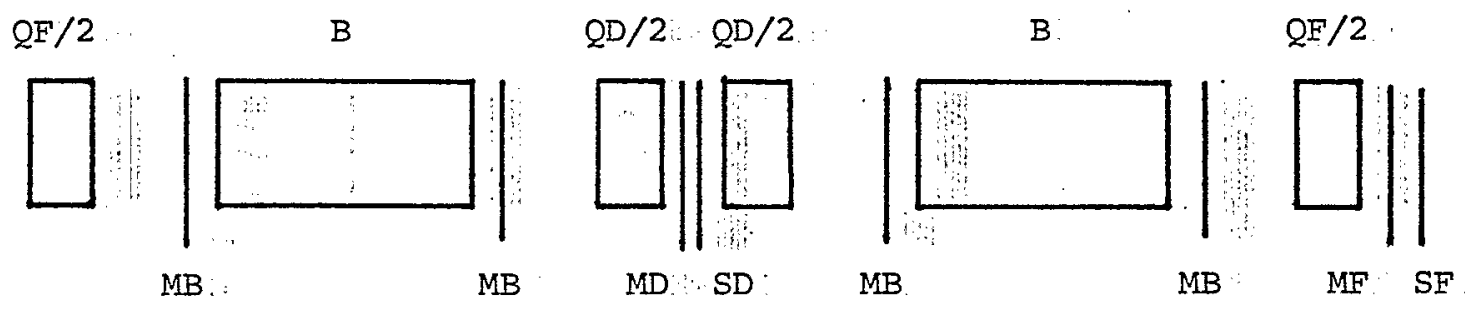

Fig. I RHIC Cell

- In this study there were no multipole fields associated with the quadrupoles. The dipole multipole coefficients $b_{n}^{\prime}$. were obtained from Pat Thompson ${ }^{1}$ and are listed in Table $I$. They are ideal coefficients based on infinite permeability iron. and do not include construction errors. The coefficients $b_{n}^{\prime}$ represent the field at $2.50 \mathrm{~cm}$ and are related to the traditional coefficients $b_{n}$ by the relation:

$$
b_{n}=b_{n}^{\prime} /(2 \cdot 5)^{n}
$$




\begin{tabular}{|c|c|c|c|}
\hline$n$ & $b_{n}^{\prime}\left(10^{-4}\right)$ & $b_{n}\left(\mathrm{~cm}^{-n}\right)$ & $b_{n}\left(m^{-n}\right)$ \\
\hline 2 & -0.02 & $-3.20 \mathrm{E}-07$ & $-3.20 \mathrm{E}-03$ \\
4 & -0.01 & $-2.56 \mathrm{E}-08$ & $-2.56 \mathrm{E}+00$ \\
6 & 0.00 & 0.00 & 0.00 \\
8 & 0.04 & $2.62 \mathrm{E}-09$ & $2.62 \mathrm{E}+07$ \\
10 & 0.08 & $8.39 \mathrm{E}-10$ & $8.39 \mathrm{E}+10$ \\
12 & -0.21 & $-3.52 \mathrm{E}-10$ & $-3.52 \mathrm{E}+14$ \\
14 & -0.84 & $-2.26 \mathrm{E}-10$ & $-2.26 \mathrm{E}+18$ \\
16 & 0.15 & $\because 6.44 \mathrm{E}-12$ & $6.44 \mathrm{E}+20$ \\
18 & 0.26 & $1.79 \mathrm{E}-12$ & $1.79 \mathrm{E}+24$ \\
\hline
\end{tabular}

Table I. Systematic multipole coefficients for the RHIC dedicated dipoles.

Tracking runs, with and without multipoles, were:made using aperture tests of $1000 \mathrm{~mm}$ (dynamic aperture) and $30 \mathrm{~mm}$ (physical aperture). The tests were made at every sextupole and multipole element- at the center of the quadrupoles and at both ends of the dipoles.

The emittances in the $x$ and $y$ directions were set equal and were $\epsilon_{x}=\epsilon_{y}=N^{2} \epsilon_{0}$ with $\epsilon_{0}=0.005$ and with $\mathrm{N}$ being the particle amplitude in PATRICIA units. For $N=1, \epsilon_{X}=\epsilon_{y}=\epsilon_{0}$ corresponds to a distance $\Delta r=\left(\Delta x^{2}+\Delta y^{2}\right)^{1 / 2}=\left(\epsilon_{0}\left(\beta_{x}+\beta_{y}\right)^{1 / 2} ;\right.$ $\Delta r$ is greatest at the quadrupole centers where $\beta=\beta=\beta_{y}:=52.0 \mathrm{~m}$ and $\beta_{y}^{F}=\beta_{x}^{D}=7.35 \mathrm{~m}$. At these positions $\Delta r=0.545 \mathrm{~mm}$ Similarly, at the ends of the dipoles $\left(\beta_{x}+\beta_{y}\right) \sim 52.7 \mathrm{~m}$ and $\Delta r=0.513 \mathrm{~mm}$. Each unit change of the PATRICIA: amplitude $\mathrm{N}$ corresponds to a radial change of the particle's amplitude of $0.545 \mathrm{~mm}$ at a quadrupole and $0.513 \mathrm{~mm}$ at either end of a dipole:

Throughout the following studies the chromaticity was set. to: zero: in both the $\mathrm{x}$ and $\mathrm{y}$ planes. The resultant dependence of tune upon momentum is shown in:, Fig $: 2$...

The results of runs made: with the $1000 \mathrm{~mm}$ aperture test and no multipoles are Iisted in Table. II. (In. Tables II to VII the quantity N(TURNS) denotes the : PATRICIA amplitude $\mathrm{N}$ and the number of turns (TURNS) the particle survived at... that amplitude. In addition, two entries appear in the column $A(\mathrm{~mm})$. The entries under $Q$ and $B$ denote the beam size at the center of the quadrupoles and at the " ends of the dipoles, respectively.) Particle trajectories were stable well beyond the magnet aperture of $30 \mathrm{~mm}$, so it was not deemed necessary to determine the actual dynamic aperture. 


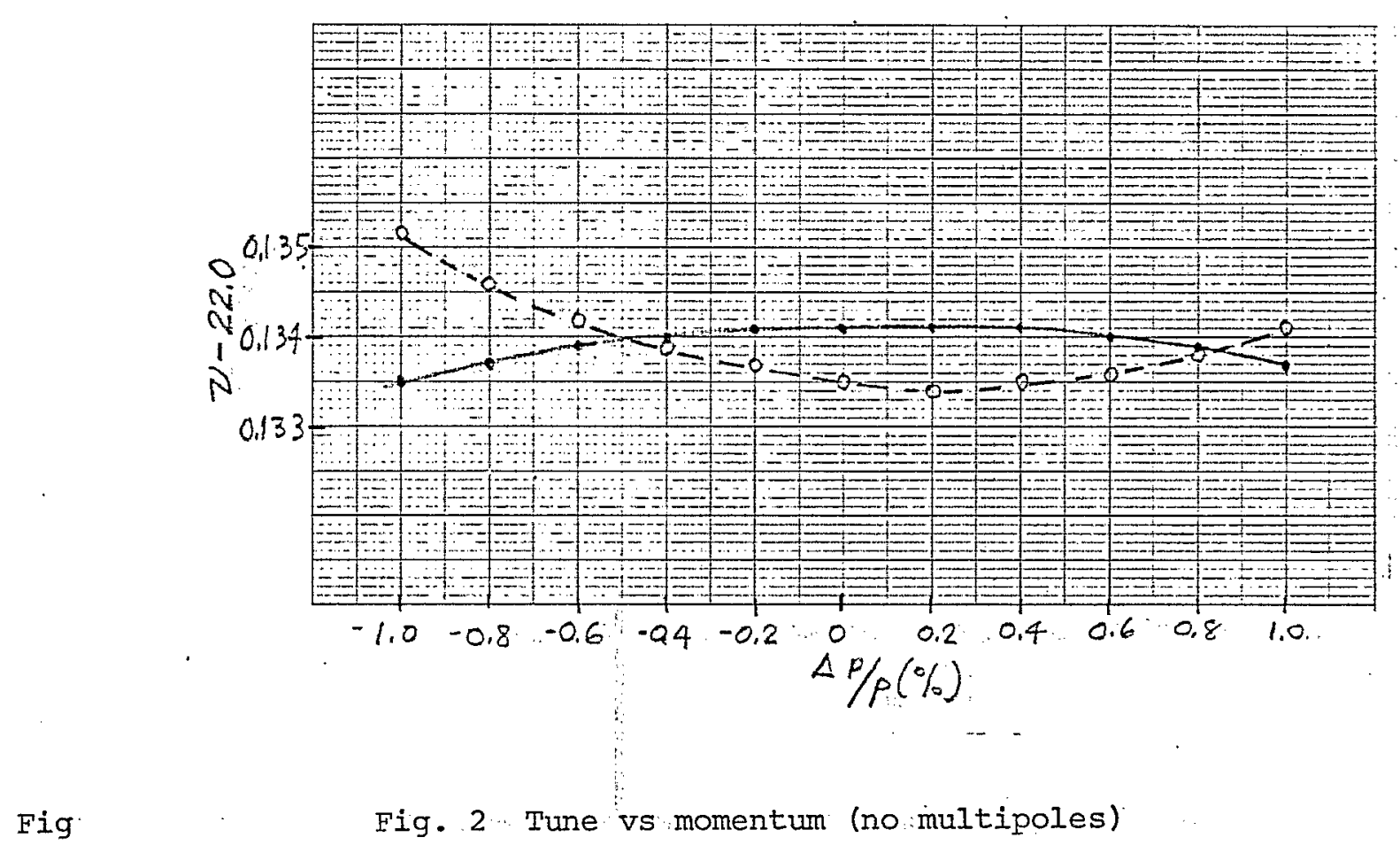

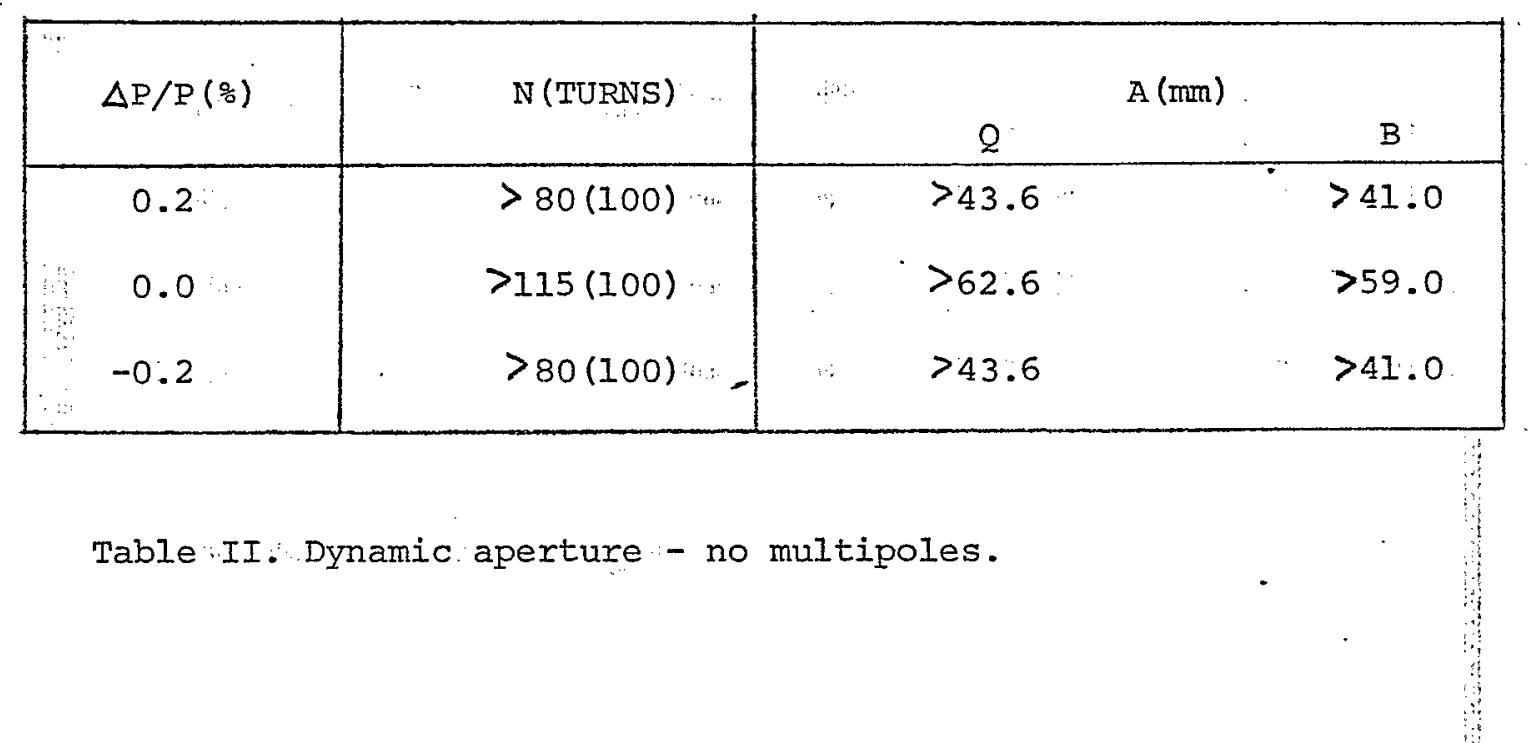

When the magnet multipoles listed in Table I. were included, the dynamic aperture was reduced. Even though a particle passed the $1000 \mathrm{~mm}$ aperture test at one: multipole element, failure was associated with amplitudes of $10^{4}$ to $10^{16} \mathrm{~mm}$ at the next multipole element only a few meters away. The results of this study appear in Table III. 


\begin{tabular}{|c|c|c|c|c|}
\hline 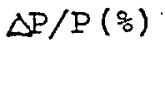 & $N(T U R N S)$ & $a^{2}$ & $A(\mathrm{~mm})$ & B \\
\hline 0.2 & $52(100), 54(4)$ & $28.3 \leq A<29.4$ & $\because$ & $26.7 \leqslant A<27.7$ \\
\hline 0.0 & $56(100), 58(6)$ & $30.5 \leqslant A \cdot \angle 31.6$ & & $28.7 \leqslant A<29.7$ \\
\hline-0.2 & $52(100), 54(69)$ & $28.3 \leq A<29.4$ & $\therefore$ & $26.7 \leqslant A .<27.7$ \\
\hline
\end{tabular}

Table III. Dynamic aperture with all multipoles:

An effort was made to identify which, if any, multipole played a dominant role. in the aperture limitation.: A series of runs was made for which one multipole; coefficient was set to zero. The column headed "without" indicates which multipole was removed. There seems to be little indication of:a "most important" multipole term. These results are listed in Table IV.

\begin{tabular}{|c|c|cc|}
\hline Without & N(TURNS) & A & A (mm) \\
\hline$b_{2}$ & $54(100), 56(90)$ & $29.4 \leq A<30.5$ & $27.7 \leq A<28.7$ \\
$b_{4}$ & $56(100), 58(6)$ & $30.5 \leq A<31.6$ & $28.7 \leq A<29.7$ \\
$b_{8}$ & $56(100), 58(7)$ & $30.5 \leq A<31.6$ & $28.7 \leq A<29.7$ \\
$b_{10}$ & $56(100), 58(7)$ & $30.5 \leq A<31.6$ & $28.7 \leq A<29.7$ \\
$b_{12}$ & $54(100), 56(30)$ & $29.4 \leq A<30.5$ & $27.7 \leq A<28.7$ \\
$b_{14}$ & $56(100), 58(17)$ & $30.5 \leq A<31.6$ & $28.7 \leq A<29.7$ \\
$b_{16}$ & $54(100), 56(67)$ & $29.4 \leq A<30.5$ & $27.7 \leq A<28.7$ \\
$b_{18}$ & $54 .(100), 56(23)$ & $29.4 \leq A<30.5$ & $27.7 \leq A<28.7$ \\
\hline
\end{tabular}

Table IV. Dependence of dynamic aperture upon one missing multipole:

Next, runs were made when half the multipole coefficients were removed; thus $b_{2}$ to $b_{10}$ or $b_{12}$ to $b_{18}$ were set equal. to zero. These results are listed in Table $V$. It is seen that removal of $b_{2}$ to $b_{10}$ has little effect while removal of $b_{12}$ to $b_{18}$ increased the dynamic aperture by $\sim 11 \mathrm{~mm}$. 


\begin{tabular}{|c|c|c|c|}
\hline Without & $N(T U R N S)$ & $\Omega$ & B \\
\hline $\mathrm{b}_{2}-\mathrm{b}_{10}$ & $54(100), 56(4)$ & $29: 4 \leq A<30.5$ & $27.7 \leq A<28.7$ \\
\hline $\mathrm{b}_{12}{ }^{-\mathrm{b}_{18}}$ & $76(100), 78(54)$ & $41: 4: \leq A<42.5$ & $39.0 \leq A<40.0$ \\
\hline
\end{tabular}

Table $V \cdot$ Dynamic aperture at $\Delta P / P=0$ with half the multipoles removed.

Further runs were made for which $\left(b_{12}\right.$ and $\left.b_{18}\right),\left(b_{14}\right.$ and $\left.b_{18}\right)$, and $\left(b_{16}\right.$ and $\left.b_{18}\right)$ were removed as well as for which $\left(b_{12}\right.$ and $\left.b_{14}\right)$ and $\left(b_{14}\right.$ and $b_{16}$ ) were removed. These results are, summarized in Table VI. With the exception of the combination $\left(\mathrm{b}_{14}\right.$ and $\left.\mathrm{b}_{18}\right)$ which increased the aperture by $\sim 4 \mathrm{~mm}$, no other combination produced much of an effect.

\begin{tabular}{|c|c|c|c|}
\hline Without & $\mathrm{N}$ (TURNS) & $\begin{array}{l}\because \\
\cdots: \\
\cdots\end{array}$ & B \\
\hline$b_{12}, b_{18}$ & $56(100), 58(4)$ & $30.5 \leq A<31.6$ & $28.7 \leq A<29.7$. \\
\hline$b_{14}, b_{18}$ & $\therefore 64(100) ; 66(8)$ & $34.9 \leqslant A<36.0$ & $32.8 \leqslant A<33.8$ \\
\hline $\mathrm{b}_{16}, \mathrm{~b}_{18}$ & $52(100), 54(19)$ & $28: 3 \leq A<29.4$ & $26.7 \leq A<27.7$ \\
\hline $\mathrm{b}_{12}, \mathrm{~b}_{14}$ & $\because 52(100) ; 54(75)$ & $28.3 \leqslant A<29.4$ & $26.7 \leqslant A<27.7$ \\
\hline$b_{14} \cdot b_{16}$ & $.54(100), 56(20)$ & $29.4 \leq A<30.5$ & $27.7 \leq A<28.7$ \\
\hline \\
\hline
\end{tabular}

Finally, runs were made using a $30 \mathrm{~mm}$ aperture test in both the quadrupoles and dipoles. The results for four different conditions: 1). No multipoles; 2). All multipoles, 3): $b_{2}$ to $b_{10}$, and 4) : $b_{12}$ to $b_{18}$ are listed in Table. VII. 


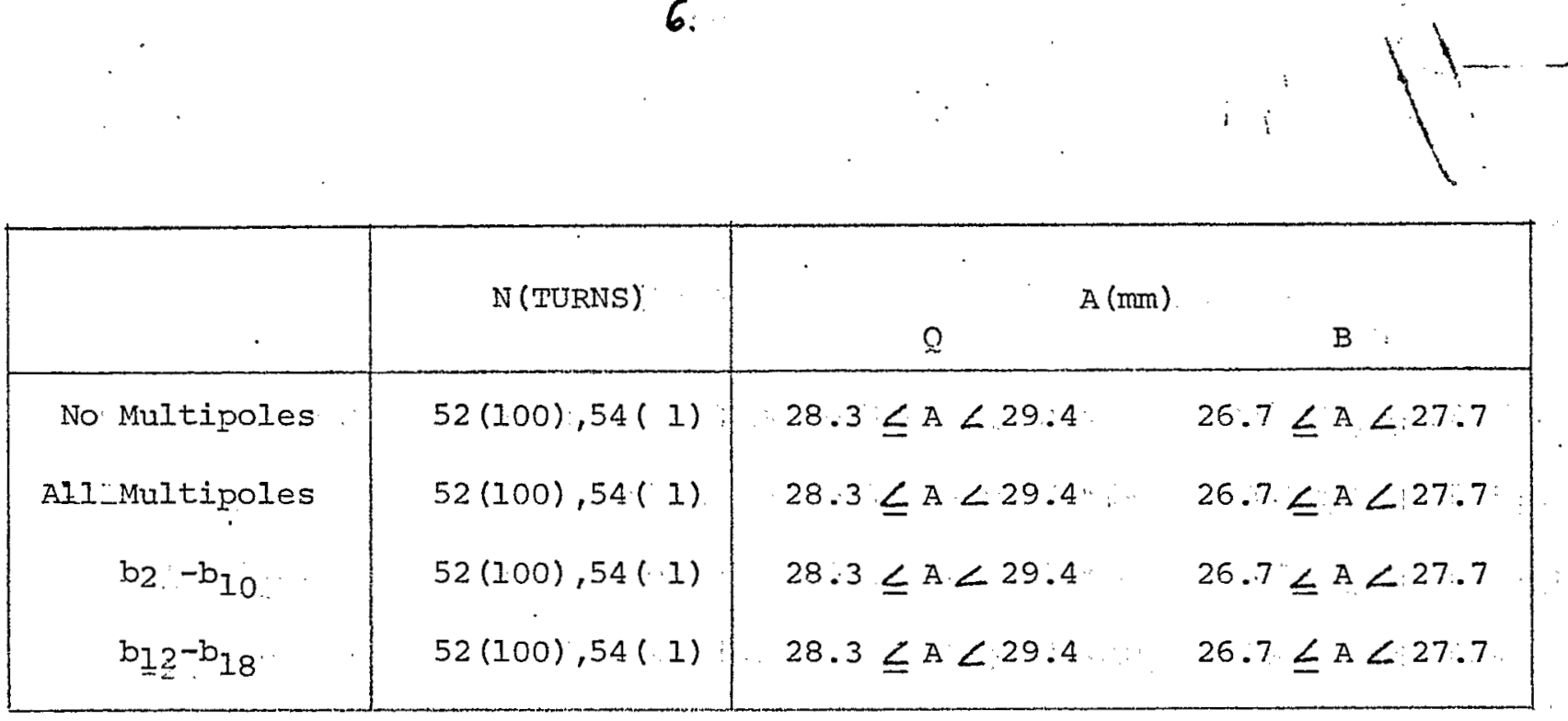

Table VII. Physical aperture (30 mm test) at $\Delta \mathrm{P} / \mathrm{P}=0$.

At the 100: turn level, the physical aperture is independent of whether or not multipoles are included. However, the dynamic aperture from Table. III is only $2 \mathrm{~mm}$ larger than the physical aperture listed in Table VII that was determined by beam size in the quadrupoles.

\section{Summary.}

The dynamic aperture without magnet multipoles wasn't fully determined but. was found to be larger than $59 \mathrm{~mm}$ in the dipoles at $\Delta P / P=0.0$.: When all systematic multipoles were included, , the dynamic aperture: in the dipoles was reduced to $28.7 \leq A<29.7 \mathrm{~mm}$. Removal of individual multipoles had little effect on the dynamic aperture; removal of $b_{12}$ to $b_{18}$ increased the dynamic aperture by $\sim 11 \mathrm{~mm}$, indicating that the higher order fields play a significant role in determining. the aperture: Removal of $\mathrm{b}_{14}$ of $\mathrm{b}_{18}$, only; increased the aperture by $4 \mathrm{~mm}$.

Finally, the physical aperture, determined by a $30 \mathrm{~mm}$ test in the dipoles. and quadrupoles, was $26.7 \leq \mathrm{A} \angle 27.7$ in the dipoles both with and without multipoles. This aperture is determined by beam size in the quadrupoles. Even so, the corresponding beam size in the dipoles is within 1 to $2 \mathrm{~mm}$ of the dynamic aperture of the dipoles. In the event that multipole coefficients for "real" dipoles are larger than those in Table $I$, the dynamic aperture rather than the physical aperture could be the limiting aperture."

\section{References ..}

1. Pat Thompson; Private communication; information derived from Magnet Note 33-16; $\operatorname{Jan} 12,(1984)$. 